\title{
Automated TEM Data Acquisition Applications Using Robotic Grid Loading
}

\author{
C. Suloway, B. Carragher, J. Pulokas, and C.S. Potter
}

National Resource for Automated Molecular Microscopy, Dept. of Cell Biology, The Scripps Research Institute, La Jolla, CA 92037

Data acquisition spanning multiple specimen grids using a TEM is conventionally done by an operator manually loading and imaging each grid. During the previous meeting we reported on using a robotic grid loading system to collect low resolution atlases for sets of 96 grids without the need for human interaction [1,2]. Here we report on new developments of the system for routinely collecting higher resolution images selected from areas of interest in the atlas.

The robotic grid loading system is integrated with Leginon, a system for automated data acquisition from a TEM [3]. High resolution data collection is carried out using two passes through a set of grids. In the first pass a low resolution atlas is acquired at a magnification of $\sim 50-100 \mathrm{X}$ so that the user can evaluate the quality of the specimen and select target areas of interest for further higher magnification. Grids are manually placed in a tray with a 96 grid capacity, and the tray is placed in the robot workspace. The robot then loads each grid and Leginon begins automated imaging according to a user defined imaging protocol. No further user interaction is required until atlases of every grid have been acquired. This typically takes 12-24 hours for a complete 96 grid tray. The user is notified by email when the imaging is completed. The user then examines the atlas and selects areas of interest on each grid to image at higher magnification (Fig. 1). Once these target areas have been selected, Leginon begins a second pass through the set of grids and no further user interaction is required. The grids are automatically reloaded, one or more low magnification images are acquired and used to automatically align the grid coordinate system between the first and second pass. High magnification images are then acquired at the user selected targets.

Routine imaging of hundreds or thousands of TEM specimens requires a very low failure rate to keep the entire system running at maximum efficiency. We have redesigned our mechanical gripper system for loading and unloading grids from the specimen holder because the reliability of the previous design was unsatisfactory for routine operation, resulting in failure rates of up to $\sim 10 \%$. The mechanical gripper was thus replaced by a new vacuum pickup system (Fig. 2) which in initial tests has reduced the failure rate to $<1 \%$.

Future goals include developing support for applications requiring more complex data collection schemes, including automated imaging of serial sections. Users will be allowed to select one or more 3-dimensional areas of interest from a low magnification atlas created during a first pass through a set of serial sections. These areas will then be imaged at high magnification during the second pass through the grids. This should largely automate the currently extremely onerous task of reconstructing 3-dimensional volumes using serial sections.

References

[1] C.S. Potter et al., Microsc. Microanal., 10 (Suppl. 2) (2004) CD 1376-1377.

[2] C.S. Potter et al., J. Struct. Bio., 146 (2004), 431-440.

[3] B. Carragher et al., J. Struct. Bio., 132 (2000), 133-145. 
[4] This research was conducted at the National Resource for Automated Molecular Microscopy which is supported by the National Institutes of Health though the National Center for Research Resources' P41 program (RR17573). The automated serial section project is supported by the National Science Foundation (DBI-0352386).

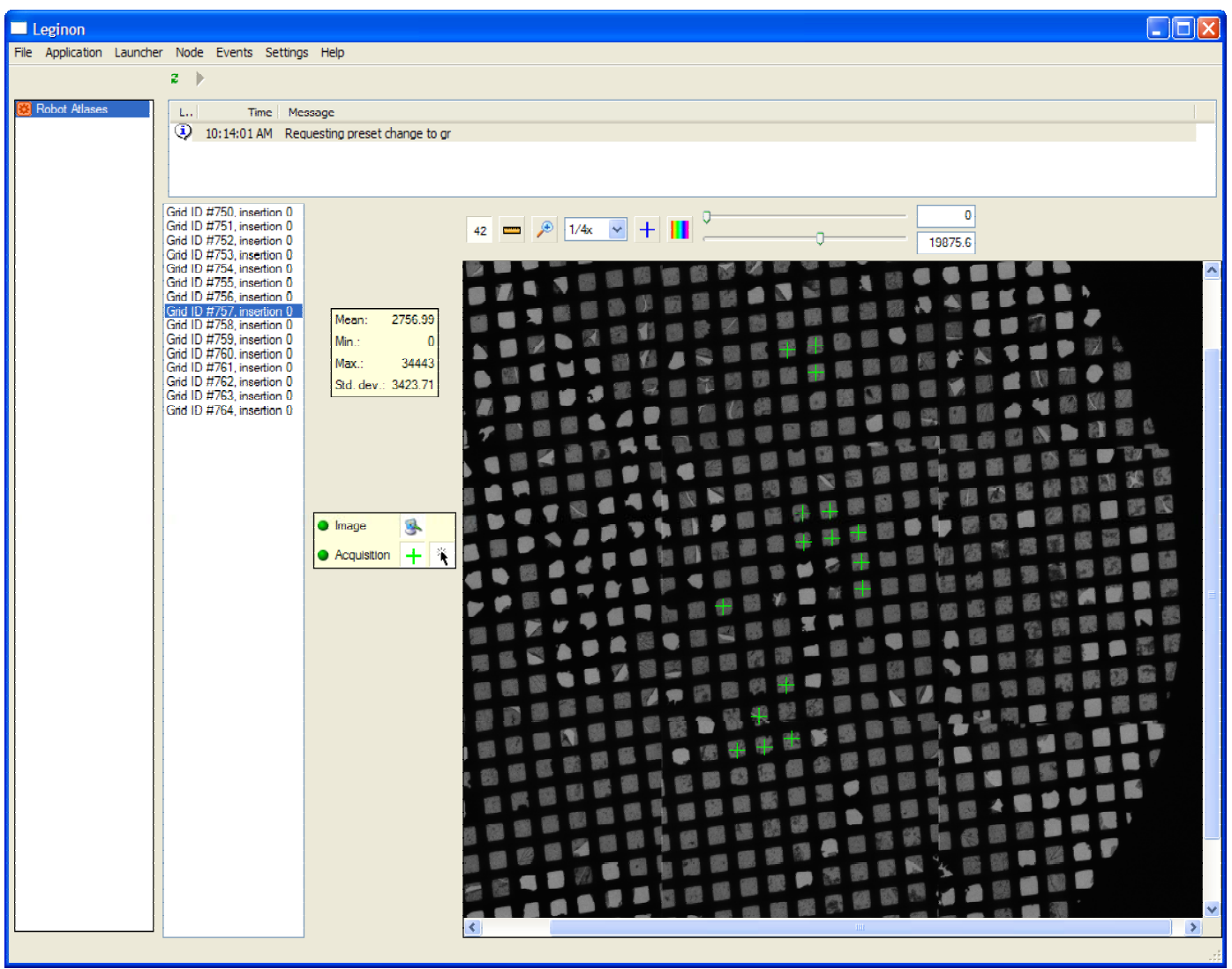

FIG. 1. User selection of targets from a low resolution atlas acquired using the Leginon system.

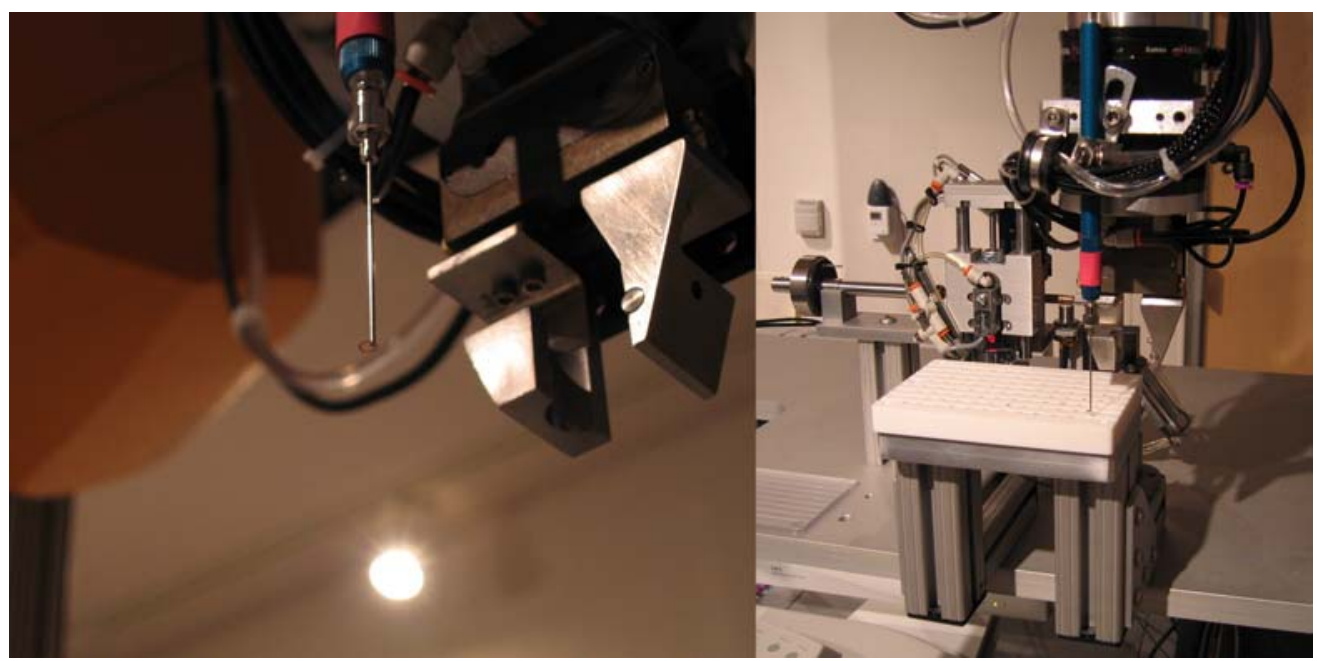

FIG. 2. Vacuum pickup system for loading grids into the specimen holder. 